\title{
Research on the Current Condition and Countermeasures of Private Colleges and Universities Student Physique Health Test under the Background of Sunshine Sports
}

\author{
Dan Zhang \\ Wuchang Shouyi University, Hubei, Wuhan,China
}

\begin{abstract}
In this paper, we conduct research on the current condition and countermeasures of private colleges and universities student physique health test under background of sunshine sports. For the further reform of basic education thought and education idea, strengthen quality education, widening specialty caliber, and promote the reform of the teaching and scientific research combined, gradually establish a notice to strengthen quality education, integrating imparting knowledge, cultivating basic ability, improve quality, develop base solid foundation, wide knowledge and strong ability and high quality specialized talented person as has the characteristics of era of diversified and comprehensive compound talents training mode and our country higher professional sports curriculum framework should be organized by the teachers, teaching conditions, teaching management, assessment, teaching reform, the teaching effect on conception to form a multi-level, multi-factor curriculum system. With this guidance, we propose the enhanced student physique health test paradigm for the private colleges and universities student that will be innovative.
\end{abstract}

Keywords- Current Condition, Countermeasures, Private, Physique Health Test, Sunshine Sports.

\section{Introduction}

Behavioral science theory, the occurrence of human behavior has the most direct relationship with the environment and health promotion model is the core content of ecology emphasizes the relationship between health behavior and the environment. Health is a fundamental right, in terms of health as the health is the concentrated reflection of health behavior. Adolescent physical health is about the future of the country and physique is the foundation of health that is the external performance of health, and sports participation is the main factor affecting the physical health [1-3].

The present comprehensive quality education ideological trend has led to a sports talk on college students' physical and mental development, but for physical education in university students' quality education production efficiency, this kind of talk more theory and practice, the real penetration to the quality of education is still a long way to go, and met with resistance. For decades, the founding of the status of school physical education never wavered in theory, but practice is frustrated, even promotes the shelf. Investigate its reason as college sports never act as the means and auxiliary tools, whether it is a moral education main body, or the knowledge center. According to the literature review, the core causes for the decrease of the college student physical health and the corresponding countermeasures could be organized as the follows. (1) Notice from sports knowledge skills to the transformation of the health belief. School physical education plays a main enlightenment for students. This effect cannot be understood narrowly to teach students the knowledge, skills, more important is to teach students knowledge, form skills at the same time, trains the student to set up the correct values of the physical health, the formation of health beliefs and behavior. (2) Pay attention to the physical health to carry out the scientific assessment. We should pay attention to set up a scientific system of physical health assessment, physical health of students in the analysis, feedback test results and provide suggestions to improve health play a role in promoting. (3) Pay 
attention to the construction of the sports culture. From sports humanities subject, the campus sports culture construction, carry out various kinds of the sports cultural activities and so on to promote college students' participation in sports activities and actively exercise and improve physical health. (4) Pay attention to the practicability of the scientific exercise. School sports education provide students exercise guidance, not only is to provide some kind of exercise or information and complex physical exercise instruction manual, and shall exercise a method combined with ascension health value of the expected, the exercise value of health is not too much time spent and the benefits of combining through strengthening exercise is expected to make the students feel the value of exercise on the development of physical and mental need of real time has high "price", which can well overcame the obstacle of participating in physical exercise behavior.

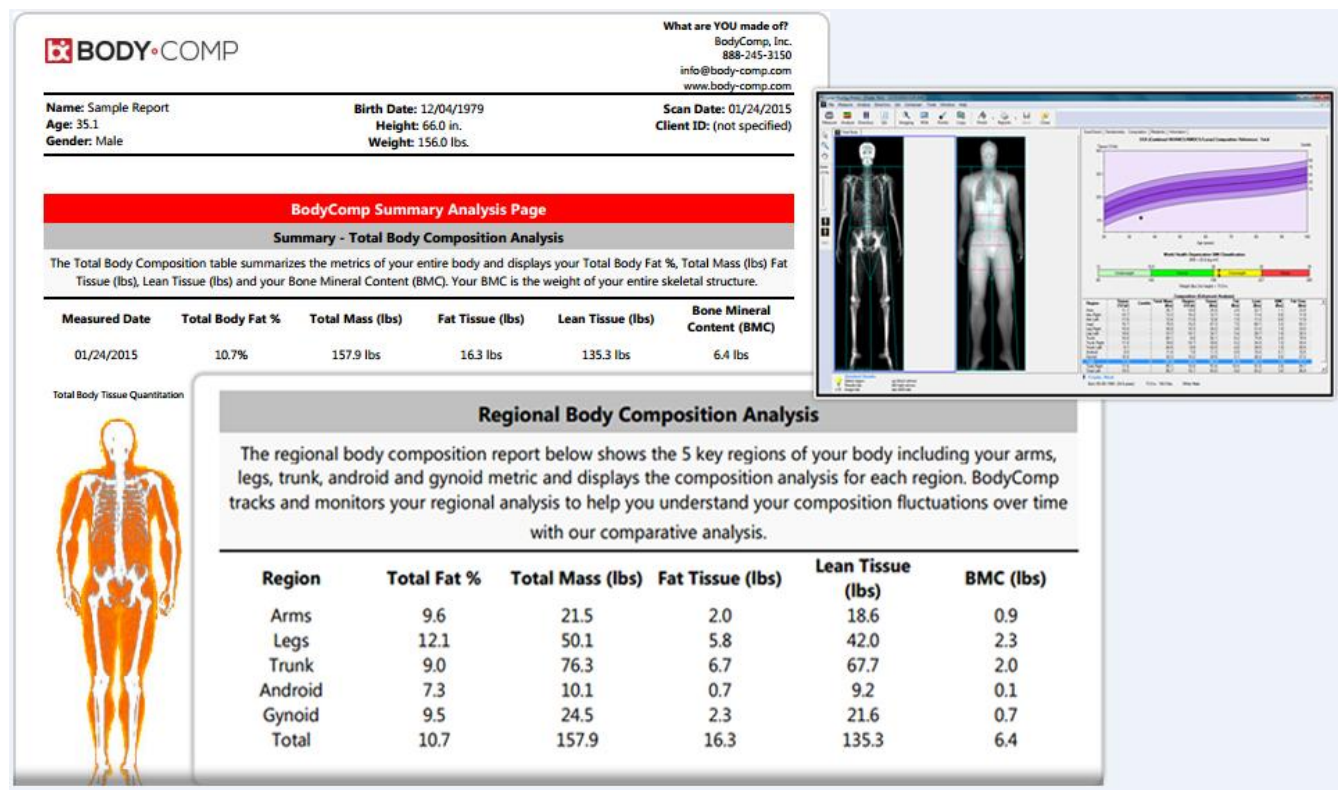

Figure 1. The Sample Report of the Student Physique Health Test

In this paper, we conduct research on the current condition and countermeasures of private colleges and universities student physique health test under the background of sunshine sports. For decades, China's physical education curriculum contents almost no changes. Although since open, try some school physical education teachers in the classroom teaching of sports to choose some new teaching content, but due to the influence of the traditional teaching ideas and the bondage of traditional habits, the new teaching content, organizational forms and teaching methods are often perish after a flash in the pan. Therefore, grasp the pulse of the times development, scientific planning and construction of ordinary university sports curriculum content system, in 21 st century is the development of Chinese higher physical education is an imminent problem.

\section{Our Proposed Methodology}

The Property of Private Colleges and Universities. In recent years, with the continuous expansion of college enrollment, the number of the private college students has been greatly rising trend and this represents a private education is more and more accepted by people, but also must realize soberly, at the same time of enrollment expansion must improve the quality of students [4].

Private colleges are to use the public fiscal funds of higher education organizations, investment in education is the basic 
characteristics of the current our country private colleges. As the modern school provided to the educatees labor products, education service is valuable. From the perspective of basic human capital, education service is a condensation on educated the sum of the knowledge, skills and the other qualities, commonly known as the human capital stock. Students education service can be converted to their knowledge, skills and other qualities, realize the use value of education service, so as to improve their own labor value. Value and use value is the two basic attributes of goods, the use value of goods to meet the needs of the people some of usefulness. The graduate of the transformation of the education service provided to the society and reap the benefits that embodies the use value of human capital stock. Use value and value both at the same time make human capital has attributes of the goods. The sustainable development of private colleges is a new topic in the field of education research and its sustainable development involves the sustainability of the internal and external environment and the policy. From the perspective of the development of private colleges, teachers are the decisive factors affecting the sustainable development. Private college teachers' sustainable relationship influences sustainability of school quality of teaching and scientific research, including the teacher introduction, stability of teachers and teachers of the sustainability of growth.

The Sunshine Sports. "Sunshine sports" is the party and government base on the adolescents declining health status and health for the future development of Chinese history to shove, situation, has the era, the pioneering significance of major decisions, to a certain extent to improve our young students physical fitness, promote the development of modern school sports, comprehensively deepen quality education produced important and profound significance [5-6].

Sports are a unity of purpose and tool is the unity of reality and the future development, from the source characteristics of the sports analysis, sunshine sports movement demanding attention to the life experience, the body needs and energy release, ultimately comes down to the life the person's life. According to the internal logic of the development of the life, namely, the order of the physical and mental development, phased, complementarity and diversity characteristics, sets up the overall view, pay attention to the continuity and quality of life. At the same time, should pay attention to the student movement changes of physiological indexes of the positive role of the body and strengthen the sports value control research, using scientific and effective method, to promote the growth and development of life. From the perspective of the structure of "sunshine sports", "sunshine sports" can be divided into material part and spirit part. Material part is the "sunshine sports" to carry out the objective of the carrier, including all kinds of sports facilities, sports venues, as well as the relevant personnel and related objects. The mental part is the abstract part, mainly including ensure smooth implementation of various rules and regulations as well as sports ideology, culture, etc.

Under this background, we should follow listed suggestion for optimizing our physical education system. (1) Build five sunshine sports development mode has the very high social value as can get reasonable scientific guarantee teenagers at each stage of development of physical exercise and sports education, to lay a solid foundation for primary lifelong physical education. (2) Five sunshine sports development model is guiding ideology, basic principles, goals, contents, policy system, management system, execution system, evaluation system, evaluation, rewards and basic punishment system and supervision system automatic error correction system sustainable development and the composition of the system and improve the sunshine sports development key.

The Student Physique Health Test. Current studies on fitness mainly in three areas: namely, 
physical anthropology, medicine and sports science as physical anthropology in constitution including morphological structure, function and metabolic characteristics, and with content of the psychological behavior characteristics. The physical health condition of students demanded by ministry of education as an important index to measure the level of higher due to college students is more, build students' physical health test center, established for students physical health test card is particularly important, should be more specialized personnel in charge of arrangement, regularly every year for all students in physical fitness test, the test results after timely feedback to the students in the department, and get published in school student physique health test overall result, as the important basis of assessment.

According to students' actual situation, formulate corresponding measures, the use of their spare time or weekend time to practice, individual training methods such as classification, to improve and enhance their physique, improve health physique test scores. Graduation, the students failed the test score according to completion of processing, due to illness or disability students treated separately, pack each semester sports test result in the student's file cover [7].

For the student physique health test enhancement, we should follow listed trend. (1) Development needs of curriculum paradigm is based on the characteristics of the students' physical and mental development stage. To be specific, the formulation must be familiar with the students in each stage of growth and development in cognition, emotion, and the characteristics of the motor skills as the American famous psychologist bloom education goal taxonomy to lay foundation for establishment of such courses. (2) Energy balance the emergence of the word more and more frequent, its role is more and more people attention. It is not only the ultimate goal of competitive sports training, also is not only used to guide the training, its purpose is to make sports population's best efforts to achieve project need body shape, body composition, and hybrid energy storage, so as to maximize movement. (3) Physical education in addition to help students learn skills related to sports as obtains the related knowledge and also can train students' correct values and attitudes, so that the students build positive, upward, and healthy life style. (4) Sports public service system is to provide the sum of product and the service behavior, including strengthening the construction of public sports facilities, and develop sports utilities and release public information, etc., to enrich the social public life and participation in social sports activities provide social security and create the conditions [8].

The Sports Educational Reform. In sports course, part of the general level of the public athletics project chosen as the material, its purpose is to develop the students body, enhance physical fitness and health, is to teach sports fitness and education methods and means, in the mind, not in accordance with the purpose of competitive sports training. Therefore, must to do a good job of the athletic sports teaching material is changed. The so-called competitive sports teaching means according to the education and sports law and law of development of human body, complex athletic sports processing and modification, refining, sublimation for the development of the body, enhance physical fitness, improve health and promote students' all-round development of body and mind of process of sports teaching material and the physical method.

College sports target should be around the basic course system, strengthen the health education of modern students, using the modern health care medical knowledge, improve the students' self-health care consciousness, make the students master and application of basic physical education and health concept and the motor skills, develop the students physical fitness; Stimulates the student sports study interest and 
hobbies, to cultivate students self-exercise habits; Carry forward the interaction of sports spirit, cultivate students overcome difficult psychological quality, positive and healthy lifestyle. To achieve goal of college physical education curriculum system, the guiding ideology of curriculum reform must establish philosophy of people-oriented education, respect and satisfy the psychological needs of students, care for students' health, scientifically curriculum design, further study of course content and system of physical education learning methods and role of the student body and mind health benefits through the course experiment research and clinical medical test, the system to sum up the experiences of the reform of college physical education curriculum, to improve the health level of college students and lifelong benefit.

The University Students' Physique. From the perspective of the ecological model of the health promotion, the external system and system similarity is the root cause of the health problems among them, and the influence of the external environment constantly infiltration to the tiny system, further influence the individual sports participation behavior [9].

Thus solve the problem of university teachers and student health must proceed from the angle of health promotion, construct the ecological environment of health promotion, the model from the point of the influence factors of exercise behavior, including the sports facilities in the campus hardware environment, health education, school sports culture atmosphere and social environment, etc. These affect the external environment of sports participation, its main performance for school sports culture atmosphere which can be called the campus social environment and basic interpersonal relationship, organization, social networks, family, dormitory, among departments such as environment and micro environment is under the influence of the campus the social environment and affects the teachers' and students' sports participation behavior. Institutions of the higher learning as a base to foster talents, shaping contemporary college students' physical health is an important goal of the ordinary university sports work, the investigations of physique monitoring software only for personal health information is stored or only statistical information, accurate statistics of the ordinary college students sub-health status and influencing factors of method mainly uses the self-designed questionnaire, cannot achieve dynamic monitoring and provide appropriate exercise prescription and fitness guidance and led to the current in this area is still in the state of relative is not sound.

\section{Conclusion}

In this paper, we conduct research on the current condition and countermeasures of private colleges and universities student physique health test under background of sunshine sports. With diversified trend of the development of physical education curriculum and the students in physical education learning behavior choice of variety, pointing to the diversity of different tendency, teaching material system to embody the two characteristics: one is for, to live, and refinement of the basic horizontal development, causes the student to have a larger degree of the freedom and initiative, and with the increasing of grade, the content of the project to reduce; second it is tend to be diversified, systematic, the longitudinal development of intelligent, can be increased with the grade to the student individual more special, to meet different ages, different interests, different levels of the students' learning needs. In the future, more corresponding research will be conducted for optimization.

\section{References}

[1] Wei, Shi. "Traditional tennis teaching to university student physique health influence and countermeasure research." Contemporary Sports Technology 7 (2013): 008. 
[2] LI, Shi-rong, and Long-long SUN. "Statistics and Analysis of North University of China Student's Physique Test Result in the 2010-2011 School Year [J]." Sports Research and Education 5 (2012).

[3] Wu, Shunwei. "Design of the Wireless Intelligent Comprehensive Physique Test System." measurement 16777216.170 (2015): 1.

[4] Zheng, Li, Tang Junling, and Zhang Tiancheng. "Comparative study of Han, Tujia and Miao students physique health status in Xiangxi." Contemporary Sports Technology 22 (2013): 003.

[5] Ping, Wang. "Analysis on the Relation between Somatotype and Physique for Students in Guangdong University of Petrochemical Technology__Based on the Physique Test Scores of 2010 Grade
Students." China Education Innovation Herald 7 (2013): 092.

[6] Sheng-jun, Z. H. O. U. "On the Obstacles to Classified Management of Physique Health Tests for College Graduates and the Countermeasures." Journal of Guangdong Peizheng College 2 (2014): 005.

[7] FENG, Li-ming, et al. "The Influences Exerted by Different Levels of BMI on College Students' Physique Health." Journal of Kaili University 6 (2012): 024.

[8] Xu-hua, Zheng. "Fuzhou Vocational School Student's Physical Health Status." Fujian Sports Science and Technology 3 (2012): 018.

[9] Jin-yan, L. I. U. "An experimental research on the influence of newly developing leisure sports-line dance in physical fitness of female college student." Liaoning Sport Science and Technology 2 (2013): 018. 